\title{
Biportal Endoscopic Spinal Surgery for Lumbar Spinal Stenosis
}

\author{
Ju-Eun Kim ${ }^{1,}$, Dae-Jung Choi ${ }^{2,}$, Eugene Jae Jin Park ${ }^{3}$, Ho-Jin Lee ${ }^{3}$, \\ Jin-Ho Hwang ${ }^{4}$, Moon-Chan Kim ${ }^{5}$, Jong-Seok $\mathrm{Oh}^{5}$ \\ ${ }^{1}$ Department of Orthopedic Surgery, Andong Medical Group Hospital, Andong, Korea \\ ${ }^{2}$ Barun Hospital, Jinju, Korea \\ ${ }^{3}$ Department of Orthopaedic Surgery, Chungnam National University School of Medicine, Daejeon, Korea \\ ${ }^{4}$ Department of Orthopaedic Surgery, CM General Hospital, Seoul, Korea \\ ${ }^{5}$ Spine Center, Himnaera Hospital, Busan, Korea
}

Biportal endoscopic spinal surgery (BESS) is a minimally invasive spinal surgery, which is basically similar to microscopic spinal surgery in terms of the use of floating technique and technically similar to conventional percutaneous endoscopic spinal surgery in terms of the use of endoscopic or arthroscopic instruments. Using two independent portals (viewing and working) and maintaining a certain distance from the bony and neural structures allow closer access to the target lesion through a panoramic view by free handling of the scope and instruments rather than through a fixed view by docking into the Kambin's triangle. Minimally invasive surgery allows for reduced dissection and inevitable muscle injury, preserving stability and reducing risks of restabilization. The purpose of fusion surgery is the same as that of the three surgical techniques stated above. Its wider range of view helps to overcome limitations of conventional endoscopic spinal surgery and to supplement the weak points of microscopic spinal surgery, such as limited working space in a tubular retractor and difficulty in accessing the contralateral area. This technique provides an alternative to unilateral or bilateral decompression of lumbar central spinal stenosis, foraminal stenosis, low-grade spondylolisthesis, and adjacent segment degeneration. Early clinical outcomes are promising despite potential for complications, such as dural tearing and postoperative epidural hematoma, similar to other procedures. Merits of BESS include decreased postoperative infection rate due to continuous irrigation throughout the procedure and decreased need for fusion surgery for one- or two-level lumbar stenosis by wide sublaminar and foraminal decompression with minimal sacrifice of stabilizing structures.

Keywords: Minimal invasive surger; Spinal stenosis; Endoscopy; Arthroscopy

\section{Introduction}

The definition of spinal stenosis originated from the Greek word stenosis. The modern description of spinal stenosis was put forth by Antoine Portal in 1803, and laminectomy was first performed by Lane for the treatment of spinal stenosis due to cauda equina syndrome at the end of the 19th century. Spinal stenosis is a common condition in the elderly ( $>60$ years of age) [1]. The conventional surgical options for spinal stenosis include open decompressive laminectomy, foraminotomy, and fusion. The current gold standard treatment for lumbar spinal stenosis refractory

Received Aug 16, 2018; Revised Sep 12, 2018; Accepted Sep 18, 2018

Corresponding author: Ju-Eun Kim

Spine Center, Himnaera Hospital, 85 Beomil-ro, Dong-gu, Busan 48735, Korea

Tel: +82-1644-9502, Fax: +82-51-710-1505, E-mail: dspfuture@hanmail.net

*Current affiliation: Spine Center, Himnaera Hospital, Busan, Korea 
to conservative treatment is facet-preserving laminectomy [2], in which a midline lumbar incision is made with the bilateral dissection of the paraspinal muscles for exposing the lumbar bony structures. However, extensive dissection results in paraspinal muscle atrophy due to ischemia and denervation $[3,4]$. In addition, resection of the posterior bone and ligaments increases the risk of complications, such as prolonged hospital stay, postoperative pain and infection, and increased blood loss [5-7]. However, the degree of bony resection required to achieve effective decompression with minimal tissue damage while reducing risks of the aforementioned complications remains debatable.

Removal of the entire lamina is not typically necessary because the main cause of spinal stenosis is related to the enlargement of the facet joint and ligamentum flavum as well as the extrusion of the intervertebral disc. At the beginning of 21 st century, several authors introduced various lumbar decompression techniques to preserve the posterior midline structures $[5,7,8]$, including unilateral laminotomy for bilateral decompression (ULBD). In ULBD, complete bilateral decompression is achieved with minimal damage to the midline structure by performing ipsilateral hemilaminotomy instead of bilateral laminectomy. This technique then evolved into microendoscopic decompression with the use of microscopy and tubular retractors [9]. For several decades, open laparotomy for appendectomy with cholecystectomy in general surgery department and open rotator cuff repair were the main procedures for the treatment of rotator cuff tears. However, laparoscopic surgery replaced open laparotomy about 10 years ago, becoming the mainstay for cancer surgeries. In addition, arthroscopy has replaced conventional open techniques for the repair of rotator cuff tears. The basic concept of biportal endoscopic spinal surgery (BESS) is similar to concepts of arthroscopic and laparoscopic surgeries, in which a scope is inserted from one side for viewing and the surgical instruments from the other side for working purposes through triangulation.

Recently, several authors have reported the use of BESS for the treatment of lumbar degenerative diseases [10-17]. BESS is advantageous because paraspinal muscle atrophy can be prevented due to reduced muscle dissection and retraction compared with open decompression and because damage to the surrounding tissues of the neural structures is low since fine discrimination under highly magnified views is possible. Decompression of the contra- lateral traversing root is relatively easy due to wide angle available for the insertion of an arthroscope. Continuous saline irrigation during surgery through the portals helps protect the soft tissue from bone debris and wash away potentially invading microorganisms to prevent infection.

Deep muscle dissection is not required when approaching the spinal canal through the interlaminar space and foramen via the intervertebral foramen. Several articles have recently described approaches for transforaminal lumbar interbody fusion, including percutaneous biportal endoscopic decompression, irrigation endoscopic decompressive laminotomy, and BESS [15]. Indications for BESS are nearly identical to those for general open spinal surgery, such as central and foraminal stenosis, adjacent segment degeneration, low-grade spondylolisthesis, and lumbar disc herniation except for high-grade spondylolisthesis, deformity correction, fracture, or pathologic conditions.

\section{Technique}

BESS can be broadly divided into interlaminar and transforaminal approaches, both of which are performed under general anesthesia with the patient in the prone position on a radiolucent frame. The basic spine instruments include a Kerrison punch and a $0^{\circ}$ or $30^{\circ} 4$ - $\mathrm{mm}$ arthro-
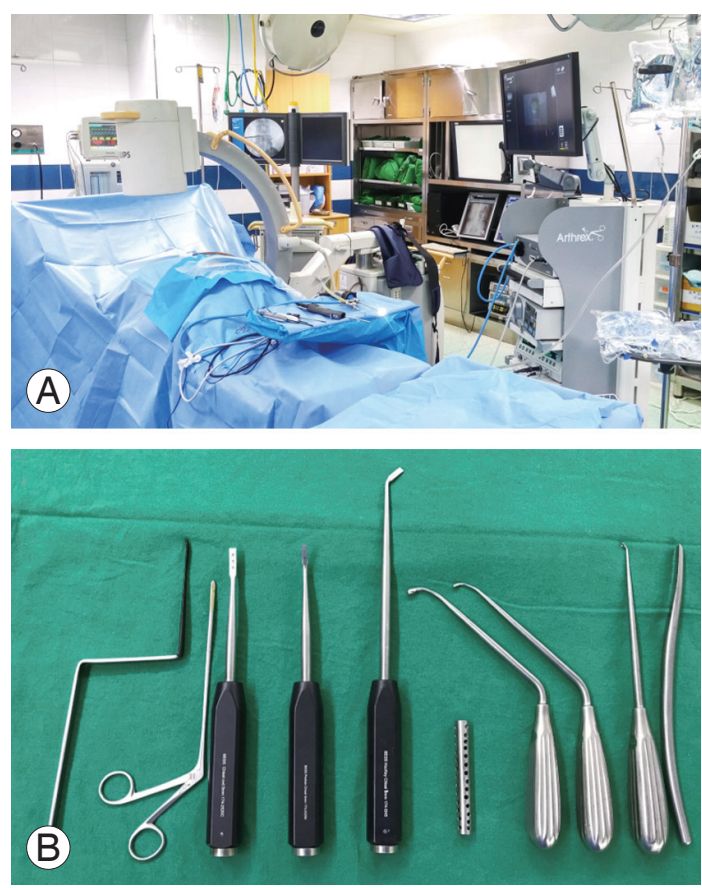

Fig. 1. (A) Set up of BESS. (B) Special instruments for BESS. BESS, biportal endoscopic spinal surgery. 
scope. Bipolar radiofrequency is used for hemostasis and an arthroscopic burr and a shaver are used to dissect and remove the bony and soft tissues (Fig. 1).

\section{Interlaminar decompression}

Level confirmation is conducted under fluoroscopic guidance before surgery. During laminotomy via the interlaminar approach, a spinal needle is inserted into the paraspinal muscle at the center of the desired surgical level. The anteroposterior and lateral views with a fluoroscope are used to confirm that the needle is located at the center of the target level. Two portals are made $1 \mathrm{~cm}$ above and $1 \mathrm{~cm}$ below the center where the needle is located and placed as close to the midline as possible near the spinous process. The distance between the two portals may differ depending on the patient's height and level, but the proximal portal is placed approximately $2 \mathrm{~cm}$ above the distal portal (Fig. 2). The incision for the portal is about $1 \mathrm{~cm}$ to introduce the arthroscope and surgical instruments. The fascia perpendicular to the skin is cut to prevent the obstruction of the water flow during surgery. Working and viewing portals can be switched during surgery at surgeon's discretion. In multilevel decompression cases, additional portals are added; for example, two levels of decompression can be performed with three portals. Isotonic saline is used to prevent water intoxication, and continuous irrigation is essential. However, excessive pressure

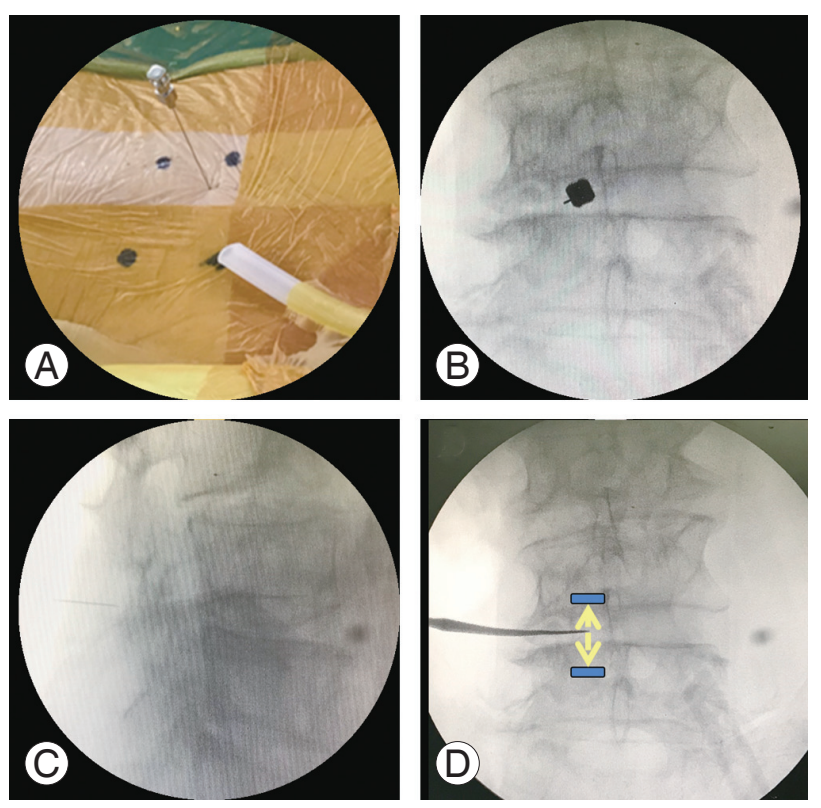

Fig. 2. (A-D) Two independent portals for the interlaminar approach. from the water pump may elevate epidural pressure and mask bleeding during infusion, which may lead to postoperative epidural hematoma after switching off the pump. It is, therefore, recommended that the water pressure be maintained below $30 \mathrm{~mm} \mathrm{Hg}$ during surgery $[10,12,13,16]$. The trocar of the scope is introduced into the viewing portal (proximal portal), and the smooth periosteal elevator is inserted into the working portal (distal portal), which are simultaneously placed at the same position of the target lamina. When triangulation is established between the arthroscope and instruments, soft tissues surrounding the interlaminar space are swept by the periosteal elevator without dissecting the paraspinal muscle. Remaining soft tissues between the lamina and ligamentum flavum are then removed using radiofrequency probes and arthroscopic shavers. When the ligamentum flavum of the target interlaminar space and half of the lower laminar are completely exposed, ipsilateral partial laminotomy is performed using an arthroscopic burr and a Kerrison punch under a magnified visual field. The ipsilateral ligamentum flavum is removed until the traversing root is completely exposed. Hemostasis for intraoperative epidural bleeding can be achieved with the use of radiofrequency probes. In case of bone bleeding, hemostasis can be achieved with the use of bone wax, as in open lumbar surgery. When ipsilateral decompression is complete, contralateral sublaminar decompression, including ligamentum flavum removal, can be easily performed owing to excellent visualization. When contralateral decompression is performed, a curette or Kerrison punch may be helpful to remove the contralateral ligamentum flavum and should be continued until the contralateral traversing root is freed. For patients with disc herniation, perform discectomy can be performed under a magnified arthroscopic view. Once the instruments and arthroscope are removed, a drain is inserted and the skin incision is closed (Fig. 3).

\section{Transforaminal decompression}

Anesthesia and position are the same as in the interlaminar approach, and a $0^{\circ}$ or $30^{\circ}$ arthroscope is used as needed. Locations of the two portal sites for the transforaminal approach are slightly different from those for the interlaminar approach. In transforaminal approach, the two portals are located approximately $1 \mathrm{~cm}$ above and below the center of the target level, near the transverse process (TP) but below the pedicle and about $2 \mathrm{~cm}$ from the ped- 

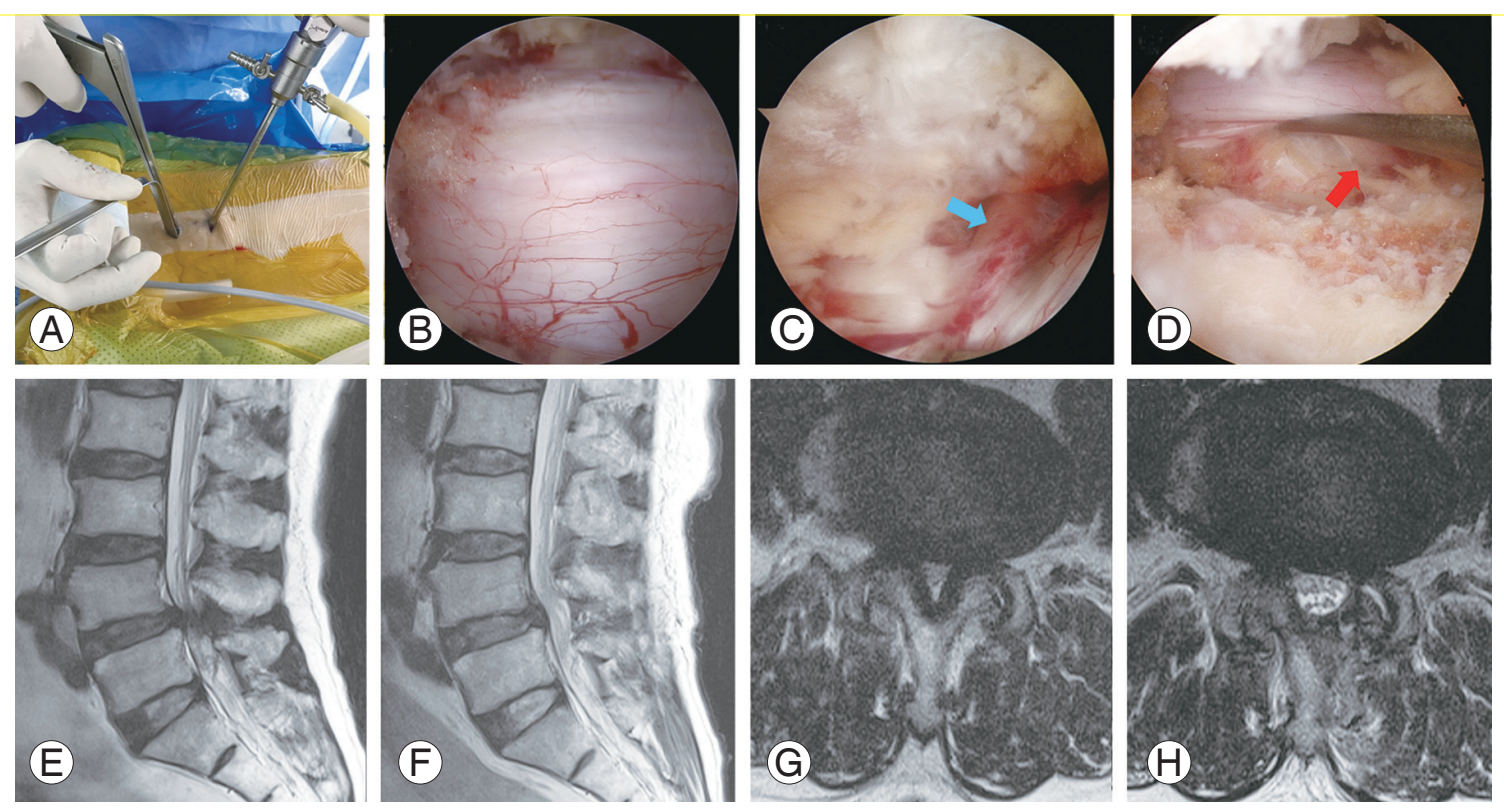

Fig. 3. (A) Intraoperative gross photo during biportal endoscopic spinal surgery. (B-D) Endoscopic image of the dura, contralateral traversing root (blue arrow), and ipsilateral traversing root (red arrow). Preoperative (E, G) and postoperative (F, H) T2-weighted magnetic resonance images of a patient who underwent interlaminar decompression for spinal stenosis at L4-L5.
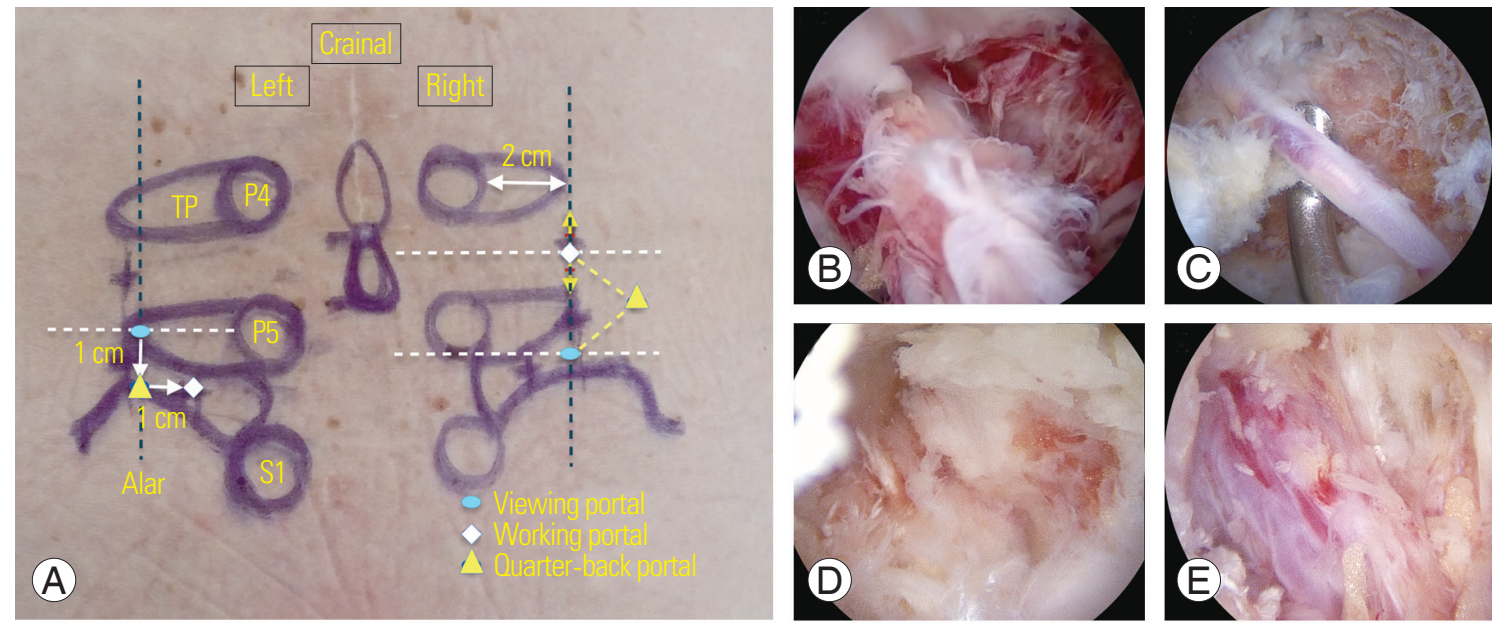

Fig. 4. (A) Portal placement for the transforaminal approach for a lumbosacral lesion. (B, C) A radical artery located above the exiting root found during transforaminal decompression. (D) An endoscopic image of partial removal of the superior articular process to allow foraminal decompression at the 12 o'clock position. (E) Endoscopic image of the exiting root after foraminal decompression. TP, transverse process.

icle lateral margin. When the transforaminal approach is applied at L5-S1 level, portal placement is slightly different. The position of the proximal portal is the same as that in surgery at other levels, but the distal portal is located $1 \mathrm{~cm}$ lateral to the sacral ala lateral border since the iliac crest hinders the insertion of the instruments. If multilevel decompression is needed, additional portals can be added. Similar to the interlaminar approach, the proximal portal is used for viewing, the distal portal is used for working, and portal switching is possible as needed during surgery. After the arthroscope is introduced into the viewing portal, a space is created between the facet and underlying TP using a periosteal elevator through the working portal under the magnified arthroscopic visual field. Muscles and tissues that interfere with the field of view within the working space are removed with a radiofrequency probe 
or shaver, and bleeding around the facet is controlled with a radiofrequency probe. Saline is infused through the viewing portal to lift the detached muscle and a smooth saline infusion is used to create a clear surgical field and view. If the outflow of the saline inside the body is poor, the visual field is drastically deteriorated and work becomes difficult. Therefore, an incision is made to the fascia of the working portal perpendicular to the skin incision to smoothen the water flow. When the arthroscope is placed on the TP in a left side transforaminal approach at L4-L5, a targeted level facet joint capsule is seen at the 12 oclock position in the arthroscopic view and the ligaments and muscles of the TP are observed at the 6 oclock position (Fig. 4). Further advancement of the arthroscope to the ventral side of the inter-TP ligament may damage the psoas muscle, and water may invade the retroperitoneal space; thus, extra attention is needed to prevent this phenomenon. Foraminal decompression starts once a proper working space is established. The hypertrophied tip of the superior articular processes appropriately removed
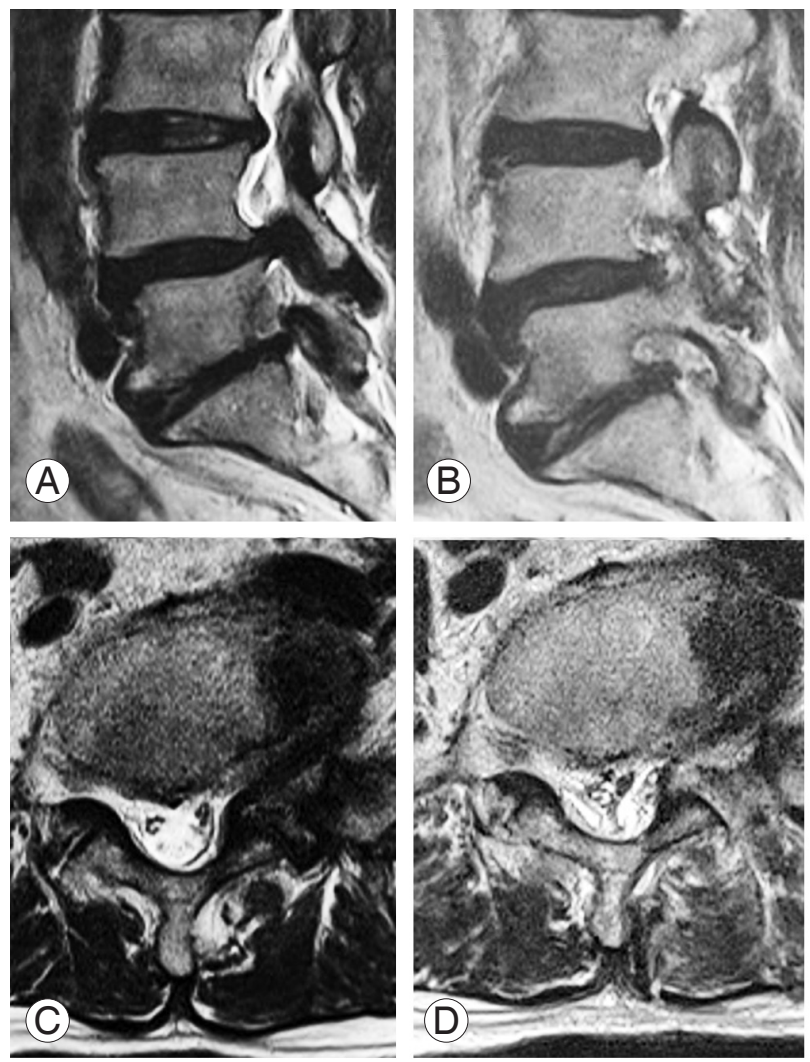

Fig. 5. Preoperative (A, C) and postoperative (B, D) T2-weighted magnetic resonance images of a patient who underwent transforaminal decompression for foraminal stenosis at L5-S1 with far lateral disc herniation. piecemeal rather than as one block with the use of a chisel or Kerrison punch. The proximal margin of the foraminal ligament is detached from the distal surface of the pedicle and TP of L4. Once the foraminal ligament covering the exiting root is completely removed, the exiting root can be observed under the radicular artery and epidural fat (Fig. 4). In patients with foraminal disc herniation, discectomy can be performed (Fig. 5).

\section{Biportal endoscopic transforaminal lumbar inter- body fusion}

The portal for biportal endoscopic transforaminal lumbar interbody fusion (BE-TLIF) is placed in the vertical position as with the two approaches mentioned above. Because the surgical target of this technique is directed at the facet joint, the portal is positioned at the pedicle above the facet joint. A transverse incision of approximately $1 \mathrm{~cm}$ is made and fascia extension is performed to allow adequate instrumentation and saline flow. The surgical procedure is similar to that of minimally invasive surgery (MIS)-TLIF, in which a tubular retractor and arthroscope are used. An arthroscopic burr, a Kerrison punch, and an osteotome are used to perform ipsilateral laminectomy, which is followed by facetectomy. After ipsilateral laminectomy and facetectomy, contralateral sublaminar decompression is performed. Bone harvested during the procedure is used for interbody bone grafting. After the ipsilateral facet removal, the transforaminal space between the exiting and traversing roots is obtained. The ligamentum flavum covering the dura and exiting nerve root are removed after the completion of ipsilateral and contralateral decompression. An incision to the intervertebral disc located in the transforaminal space between the exiting and traversing roots is made with the use of a radiofrequency probe or arthroscopic knife. Then, discectomy is performed using pituitary forceps and a special curette. After the arthroscope is introduced into the disc space, the cartilaginous endplate is removed to expose the subchondral bone while looking directly at the disc space under a magnified arthroscopic field. Autologous bone is grafted through a specialized cannula during interbody bone grafting due to the concern of bone loss caused by continuous irrigation. After bone grafting is performed, a cage is inserted. Two small retractors protect the exiting and traversing roots to prevent damage during cage insertion (Fig. 6). When the procedure is completed, a percutaneous pedicle screw 

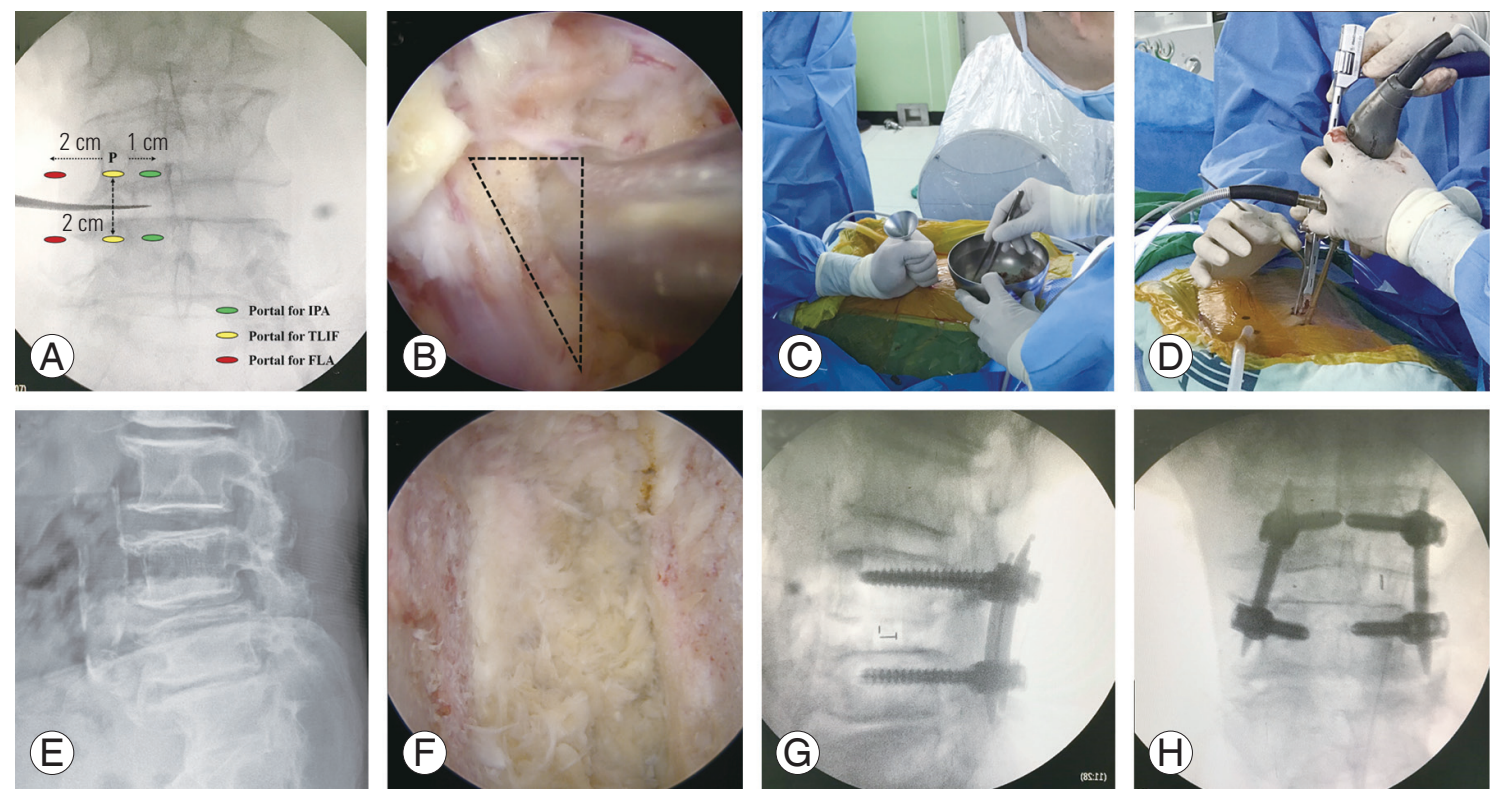

Fig. 6. (A) Portal locations of three different biportal endoscopic approaches. (B) Space between traversing and exiting root for cage insertion. (C) Intraoperative photograph. Fluoroscopy was used when bone graft procedure was conducted. (D) Once the cage is inserted, two semi-tubular retractors protect the traversing and exiting roots. (E) Preoperative plain radiograph of a patient with spondylolisthesis at L4-L5. (F) Endoscopic image showing the intervertebral disc space with complete removal of the cartilaginous endplate. (G, H) Intraoperative anteroposterior and lateral fluoroscopic views showing reduction of spondylolisthesis at $L 4-L 5$ and the position of the cage. IPA, ipsilateral posterior approach; TLIF, transforaminal lumbar interbody fusion; FLA, far lateral approach.

is fixed in the same manner as in MIS-TLIF. Two percutaneous pedicle screws on the ipsilateral side are inserted through two previously used portals, and two percutaneous pedicle screws are inserted through a new incision on the contralateral side.

\section{Outcome and Complications}

There has not yet been a prospective comparison of clinical outcomes among BESS and other decompression techniques, but most articles introducing this technique have reported favorable clinical outcomes and minimal complications $[10,12-16,18]$. In a series with a mean follow-up of 28 months following BESS and interlaminar decompression in 94 patients diagnosed with spinal stenosis, the Macnab criteria showed a good or better outcome in $87 \%$ of the patients and the Oswestry Disability Index (ODI) improved from $64.2 \pm 10.0$ to $23.0 \pm 20.8$. In this study, dural tearing occurred in six cases, which was repaired by conservative treatment without any additional procedure [16]. In another study of BESS and interlaminar decompression in 58 patients with spinal stenosis, the outcome was good or better in $81 \%$ of the patients. Eight patients experienced complications related to surgery, with one case of postoperative epidural hematoma, two cases of dural tearing, two cases of transient leg numbness, and three cases of postoperative headache [12]. In a study of BESS and interlaminar decompression in 105 patients with spinal stenosis using a $30^{\circ}$ arthroscope, ODI improved from $67.4 \pm 11.5$ to $22.9 \pm 12.4$, with two cases of dura tearing and one case of postoperative epidural hematoma [13]. In all three studies, perioperative complications occurred at the beginning of the learning curve and no wound infection occurred. Although this study did not focus on spinal stenosis, a comparison of patients who underwent discectomy with BESS and open decompression demonstrated that the early postoperative Visual Analog Scale (VAS) score was superior in discectomy using the BESS technique compared with that in open decompression and was advantageous in terms of estimated blood loss and hospital stay. However, there was no significant difference in ODI and VAS score between the two approaches [18].

To date, two technical notes have been published regarding the use of BESS with transforaminal approach, but no randomized controlled study has been performed. Transforaminal decompression in 21 patients with foraminal stenosis with a follow-up period of 14.8 months showed that leg VAS score improved from $7.5 \pm 0.9$ to 
$2.5 \pm 1.2$ and the Macnab criteria showed good or better outcome in $81 \%$ of the patients. One case of dura tear was also observed in this study [10]. In another study of transforaminal decompression using another BESS technique, although there were only 12 cases and a short follow-up period, no complications of dural tearing or neurological damage occurred [14]. BESS with transforaminal approach follows the same principle as facet-preserving microscopic foraminotomy, in which less than $50 \%$ of the superior articular process is removed. The superior articular processes can be resected based on the upper border of $\mathrm{TP}$ at the lower level of the vertebra. Although there has been no report on spinal instability following the removal of the facet joint, translational and rotational stability is achieved in the movable segment when more than $50 \%$ of the joint is removed [19]. BESS with transforaminal approach seems to prevent spinal instability by preserving more than $50 \%$ of the facet joint. It is performed with an arthroscopic magnified view using continuous saline irrigation such that surgeons can visualize the pathology more clearly.

There have been only two articles reporting technical notes and clinical outcomes of lumbar interbody fusion through BESS $[15,20]$. In a study of 69 patients who underwent biportal endoscopic lumbar fusion, the followup period was not longer than 1 year, but VAS score improved from $8.12 \pm 0.63$ to $2.79 \pm 1.24$ and ODI improved significantly from $45.65 \pm 12.97$ to $15.41 \pm 9.07$. Perioperative complications occurred in five cases, with two cases of dural tearing and three cases of postoperative epidural hematoma. Recent studies of BE-TLIF have reported that TLIF is possible during BESS, but dural tearing and root injuries occurred as perioperative complications; thus, caution is required to avoid root damage during cage insertion.

In case of lateral and anterior lumbar interbody fusion, restoration of sagittal balance and indirect decompression of spinal stenosis can be achieved through disc height restoration and spondylolisthesis reduction, but there is a disadvantage that canal encroachment cannot be directly restored in cases with severe spinal stenosis. Although TLIF through a direct posterior approach enables direct decompression of the spinal canal, it may damage the posterior muscles and ligamentous structures, leading paraspinal muscles atrophy. Direct decompression of the spinal canal is possible with BE-TLIF using BESS while preventing muscle atrophy due to less muscle dissection and retraction. In addition, endplate preparation can be performed thoroughly through a magnified arthroscopic view, which is advantageous for interbody fusion.

According to Choi et al. [21], the learning curve of BESS is relatively short and the complication rate in the early learning period is approximately $10.3 \%$. Dural and root injuries and postoperative epidural hematoma occurred as complications with this new technique [21]. To date, there have been 648 patients who underwent interlaminar decompression, transforaminal decompression, and interbody fusion through BESS in 14 articles related to the biportal endoscopic technique and lumbar spine in the PubMed database (https://www.ncbi.nlm.nih.gov/ pubmed). However, complications occurred in 39 cases (6.0\%), with 20 cases of dural tearing, which was the most common complications during BESS surgery. Postoperative headache occurred in three cases, postoperative hematoma in five, root injury in four, transient paresthesia in two, and postoperative incomplete decompression in four. One case of iatrogenic hydroperitoneum occurred in the retroperitoneal space filled with water after the ventral approach to the psoas muscle during transforaminal decompression.

One of the noteworthy features of BESS is that postoperative infection, a relatively common complication of conventional lumbar spinal surgery, has not been reported. In a review article, postoperative infection of the lumbar spine occurred in $1 \%$ of the patients who underwent open lumbar discectomy and $1.9 \%-4.4 \%$ of those who underwent lumbar fusion surgery [22,23]. The incidence of infection following spinal surgery is lower than that with MIS [24]. However, there has been no high-quality randomized controlled study or systemic review regarding infection rates between conventional open spinal surgery and BESS; thus, evidence to suggest that the risk of spinal infection is reduced with BESS remains insufficient. However, a review of 14 articles related to the biportal endoscopic technique reported no instance of spinal infection in 648 patients who underwent BESS. One of the advantages of BESS is that continuous irrigation with saline is used at a greater frequency than with conventional open spinal surgery. Because of the various reasons mentioned above, we speculate that BESS may reduce the risk of spinal infection.

The overall complication rate of BESS is close to $6 \%$, which is relatively high and most complications were root injury, dural tearing, and incomplete decompression. Tur- 
bid water and an obscure field due to failure of bleeding control and continuous irrigation may lead to the abovementioned complications for surgeons not familiar with the technique and visual field. However, an attempt to increase the saline pressure to control bleeding should be avoided because of the risk of increases intracranial pressure and delayed recovery from postoperative headache or general anesthesia [21]. Rather than attempting to obtain a clear view by increasing the infusion pressure, it seems more sensible to attempt facilitating outflow by applying an extension or cross cut of the fascia incision through the working portal. A clear visual field obtained through smooth continuous irrigation can prevent the complications mentioned here.

Several articles have reported the advantages of BESS. First, dissection of the paraspinal muscle is minimized, resulting in less damage to the posterior ligament and soft tissue and less need for inevitable fusion surgery for oneor two-level spinal stenosis with stability. Second, minimally invasive spinal surgery, such as microscopic ULBD, has the advantage of preserving the facet and contralateral bony structures compared with conventional laminectomy, but incomplete decompression may occur due to a restricted visual field and movement of the instruments. However, BESS has the advantage of microscopic ULBD to achieve complete decompression under a magnified arthroscopic field without the restriction of instrument movement because of the use of an independent portal. Third, continuous saline irrigation during surgery is a great advantage for preventing infection as well as ensuring a clean field of view.

\section{Conclusions}

BESS is a minimally invasive spinal surgery for the direct decompression of neural elements with dynamic handling of instruments under a clear view while reducing muscle dissection and damage to the posterior lumbar structures. It can be considered as the next generation of surgical development after open lumbar surgery and microscopic spinal surgery, with results comparable to the results of those surgical techniques other than conventional endoscopic spinal surgery with the use one portal. Its technical identity to open spinal surgery comes from the floating technique under triangulation. BESS is not a totally new procedure but rather an innovative use of an endoscope or arthroscope in spinal surgery. A little difference can make a big difference in the outcome of spinal surgery.

\section{Conflict of Interest}

No potential conflict of interest relevant to this article was reported.

\section{References}

1. Weinstein JN, Lurie JD, Tosteson TD, et al. Surgical versus nonsurgical treatment for lumbar degenerative spondylolisthesis. N Engl J Med 2007;356:2257-70.

2. Gibson JN, Waddell G. Surgery for degenerative lumbar spondylosis. Cochrane Database Syst Rev 2005;(4):CD001352.

3. Hu ZJ, Fang XQ, Zhou ZJ, Wang JY, Zhao FD, Fan SW. Effect and possible mechanism of musclesplitting approach on multifidus muscle injury and atrophy after posterior lumbar spine surgery. J Bone Joint Surg Am 2013;95:e192

4. Sihvonen T, Herno A, Paljarvi L, Airaksinen O, Partanen J, Tapaninaho A. Local denervation atrophy of paraspinal muscles in postoperative failed back syndrome. Spine (Phila Pa 1976) 1993;18:575-81.

5. Celik SE, Celik S, Goksu K, Kara A, Ince I. Microdecompressive laminatomy with a 5-year follow-up period for severe lumbar spinal stenosis. J Spinal Disord Tech 2010;23:229-35.

6. Postacchini F, Cinotti G, Perugia D, Gumina S. The surgical treatment of central lumbar stenosis: multiple laminotomy compared with total laminectomy. J Bone Joint Surg Br 1993;75:386-92.

7. Thome C, Zevgaridis D, Leheta O, et al. Outcome after less-invasive decompression of lumbar spinal stenosis: a randomized comparison of unilateral laminotomy, bilateral laminotomy, and laminectomy. J Neurosurg Spine 2005;3:129-41.

8. Mikami Y, Nagae M, Ikeda T, Tonomura H, Fujiwara $\mathrm{H}$, Kubo T. Tubular surgery with the assistance of endoscopic surgery via midline approach for lumbar spinal canal stenosis: a technical note. Eur Spine J 2013;22:2105-12.

9. Pao JL, Chen WC, Chen PQ. Clinical outcomes of microendoscopic decompressive laminotomy for degenerative lumbar spinal stenosis. Eur Spine J 2009;18:672-8. 
10. Ahn JS, Lee HJ, Choi DJ, Lee KY, Hwang SJ. Extraforaminal approach of biportal endoscopic spinal surgery: a new endoscopic technique for transforaminal decompression and discectomy. J Neurosurg Spine 2018;28:492-8.

11. Choi DJ, Kim JE, Jung JT, et al. Biportal endoscopic spine surgery for various foraminal lesions at the lumbosacral lesion. Asian Spine J 2018;12:569-73.

12. Eum JH, Heo DH, Son SK, Park CK. Percutaneous biportal endoscopic decompression for lumbar spinal stenosis: a technical note and preliminary clinical results. J Neurosurg Spine 2016;24:602-7.

13. Kim JE, Choi DJ. Unilateral biportal endoscopic decompression by $30^{\circ}$ endoscopy in lumbar spinal stenosis: technical note and preliminary report. J Orthop 2018;15:366-71.

14. Kim JE, Choi DJ. Bi-portal arthroscopic spinal surgery (BASS) with $30^{\circ}$ arthroscopy for far lateral approach of L5-S1: technical note. J Orthop 2018;15:354-8

15. Kim JE, Choi DJ. Biportal Endoscopic transforaminal lumbar interbody fusion with arthroscopy. Clin Orthop Surg 2018;10:248-52.

16. Soliman HM. Irrigation endoscopic decompressive laminotomy: a new endoscopic approach for spinal stenosis decompression. Spine J 2015;15:2282-9.

17. Torudom Y, Dilokhuttakarn T. Two portal percutaneous endoscopic decompression for lumbar spinal stenosis: preliminary study. Asian Spine J 2016;10:33542.
18. Kim SK, Kang SS, Hong YH, Park SW, Lee SC. Clinical comparison of unilateral biportal endoscopic technique versus open microdiscectomy for singlelevel lumbar discectomy: a multicenter, retrospective analysis. J Orthop Surg Res 2018;13:22.

19. Teo EC, Lee KK, Qiu TX, Ng HW, Yang K. The biomechanics of lumbar graded facetectomy under anterior-shear load. IEEE Trans Biomed Eng 2004;51:443-9.

20. Heo DH, Son SK, Eum JH, Park CK. Fully endoscopic lumbar interbody fusion using a percutaneous unilateral biportal endoscopic technique: technical note and preliminary clinical results. Neurosurg Focus 2017;43:E8.

21. Choi DJ, Choi CM, Jung JT, Lee SJ, Kim YS. Learning curve associated with complications in biportal endoscopic spinal surgery: challenges and strategies. Asian Spine J 2016;10:624-9.

22. Weinstein MA, McCabe JP, Cammisa FP Jr. Postoperative spinal wound infection: a review of 2,391 consecutive index procedures. J Spinal Disord 2000;13:422-6.

23. Fang A, Hu SS, Endres N, Bradford DS. Risk factors for infection after spinal surgery. Spine (Phila $\mathrm{Pa}$ 1976) 2005;30:1460-5.

24. Meredith DS, Kepler CK, Huang RC, Brause BD, Boachie-Adjei O. Postoperative infections of the lumbar spine: presentation and management. Int Orthop 2012;36:439-44. 